Cite this: J. Mater. Chem. B, 2014, 2 , 3626

Received 4th February 2014

Accepted 26th March 2014

DOI: $10.1039 / c 4 t b 00184 b$

www.rsc.org/MaterialsB

\section{Multivalent grafting of hyperbranched oligo- and polyglycerols shielding rough membranes to mediate hemocompatibility $\dagger$}

\author{
Axel T. Neffe, $t^{\text {tabc }}$ Maik von Ruesten-Lange, $t^{\text {ab }}$ Steffen Braune, $t^{\text {ab }}$ Karola Lützow, \\ Toralf Roch, ${ }^{\text {ac }}$ Klaus Richau, ${ }^{a}$ Anne Krüger, ${ }^{a}$ Tobias Becherer, ${ }^{c d}$ \\ Andreas F. Thünemann, ${ }^{e}$ Friedrich Jung, ${ }^{\text {ac }}$ Rainer Haag ${ }^{\text {cd }}$ and Andreas Lendlein ${ }^{\star a b c d}$
}

Hemocompatible materials are needed for internal and extracorporeal biomedical applications, which should be realizable by reducing protein and thrombocyte adhesion to such materials. Polyethers have been demonstrated to be highly efficient in this respect on smooth surfaces. Here, we investigate the grafting of oligo- and polyglycerols to rough poly(ether imide) membranes as a polymer relevant to biomedical applications and show the reduction of protein and thrombocyte adhesion as well as thrombocyte activation. It could be demonstrated that, by performing surface grafting with oligo- and polyglycerols of relatively high polydispersity (>1.5) and several reactive groups for surface anchoring, full surface shielding can be reached, which leads to reduced protein adsorption of albumin and fibrinogen. In addition, adherent thrombocytes were not activated. This could be clearly shown by immunostaining adherent proteins and analyzing the thrombocyte covered area. The presented work provides an important strategy for the development of application relevant hemocompatible 3D structured materials.

\section{Introduction}

Highly hydrophilic polymers such as oligo- and poly(ethylene glycol)s (OEG, PEG) and zwitterionic structures such as phosphorylcholine-, carboxybetaine- and sulfobetaine-based compounds have been investigated intensively for surface functionalization, ${ }^{\mathbf{1 , 2}}$ as they show very promising results by reducing protein and cell adhesion in ideally flat model surfaces such as gold or glass, ${ }^{3,4}$ when grafted on smooth polymers, ${ }^{5}$ and as particle coatings. ${ }^{6,7}$ As cell adhesion in many cases is protein mediated, lower protein adsorption simultaneously might lead to lower cell adhesion. Protein adsorption is reduced with increasing grafting density and with increasing PEG chain length. However, the grafting density generally decreases with the chain length. ${ }^{8-10}$ Another interesting observation is the quite different performance of grafted linear and star-shaped PEGs. ${ }^{11}$ It could be shown that linear PEGs are obviously more effective

anstitute of Biomaterial Science and Berlin-Brandenburg Center for Regenerative Therapies, Helmholtz-Zentrum Geesthacht, Kantstrasse 55, 14513 Teltow, Germany. E-mail: axel.neffe@hzg.de; andreas.lendlein@hzg.de

${ }^{b}$ Institute of Chemistry, University of Potsdam, Germany

${ }^{c}$ Helmholtz Virtual Institute - Multifunctional Biomaterials for Medicine, Teltow and Berlin, Germany

${ }^{d}$ Institute of Chemistry and Biochemistry, Freie Universität Berlin, Germany

${ }^{e} B A M$ Federal Institute for Materials Research and Testing, Germany

$\dagger$ Electronic supplementary information (ESI) available. See DOI: 10.1039/c4tb00184b

\$ These authors contributed equally. in shielding a smooth surface and reducing protein adsorption than branched derivatives, which was related to the positioning of the center of mass of the grafted chains. If this center is too far above the surface, any protein passing the PEG chain acting as a diffusion barrier will be trapped below the shield.

These experiences from smooth surfaces do not appear to be transferable, however, to rough surfaces, which may have higher relevance in certain applications such as membranes in biomedicine. This is because 3D and not 2D structures have to be functionalized, which additionally have a lower number of reactive groups per surface area compared to gold or glass. In fact, in previously reported experiments, shorter OEGs covalently grafted onto the surface of poly(ether imide) (PEI) membranes resulted in better surface shielding than the larger PEGs. ${ }^{12}$ Though the adsorption of albumin could be reduced, there was no strong influence on thrombocyte adhesion by surface functionalization, and no difference between $-\mathrm{OH}$ or -OMe terminated species was found. ${ }^{\mathbf{1 2}}$ Grafted linear, sidechain methylated oligoglycerols behaved similarly. ${ }^{13}$ In the literature, often only the reduction of albumin adsorption after membrane functionalization was reported, with results similar to the ones described above, ${ }^{\mathbf{1 4}}$ which indicates that albumin is an inadequate predictor for the different but also interlinked adhesion patterns of blood plasma proteins. ${ }^{15}$ We hypothesized that branched oligo- or polyethers with several functional groups for potential coupling to the surface can be effective for surface functionalization of rough surfaces by multivalent attachment of the oligo- and polyethers. In this way, a better 
surface shielding and stable spreading of the oligoethers on the surface was targeted, simultaneously shifting the center of mass closer to the surface than has been reported for branched PEGs. ${ }^{11}$ Furthermore, we hypothesized that functionalization with molecules of different sizes yields a better surface shielding than with polymers of low polydispersity, like tiling a floor with polygons of different sizes and shapes.

Therefore, hyperbranched oligoglycerols (hOG) and polyglycerols (hPG) ${ }^{\mathbf{1 6}}$ were selected as polyethers for functionalization of PEI membranes. PEI has high potential for biomedical applications, as it is non-toxic, steam sterilizable, and moreover can be functionalized by nucleophiles under aqueous conditions. hOG and hPG have been shown to be more resistant to heat $^{17,18}$ and oxidation ${ }^{13}$ than OEG and PEG, as well as they resist protein adsorption equally well. ${ }^{19}$ The synthetic access to hOG and hPG with a polydispersity above 1.5 (ref. 20) allows variation of the molecular weight as well as the end group functionality, two important aspects for protein resistant properties of the functionalized surfaces.

In the following, the formation of PEI membranes and their surface functionalization with several aminated hOG and hPG will be described and compared to non-functionalized PEI-0, alkaline treated PEI pH 11 and a PEI membrane functionalized with a linear, side chain methylated OG (OG-OMe). The end group functionalities ( $\mathrm{OH}$ or OMe) of hOG $\left(M_{\mathrm{n}}=2000 \mathrm{~g} \mathrm{~mol}^{-1}\right)$ and hPG $\left(M_{\mathrm{n}}=5000 \mathrm{~g} \mathrm{~mol}^{-1}\right)$ were varied, and their solution structures were studied by small angle X-ray scattering (SAXS). The surface functionalization was determined by FT-ATR-IR and XPS, while shielding of the bulk material surface through functionalization was investigated by contact angle measurements and streaming potential measurements. Functionalized PEI surfaces are known to be structured in three layers, the top layer of the introduced group, an intermediate layer formed by free carboxylic acid functions produced during the surface functionalization, and the bulk polymer. Protein adsorption on the membranes was quantified in an indirect bicinchoninic acid (BCA) assay as well as investigated by direct immunostaining after contact with blood plasma. The adhesion of human thrombocytes was visualized and quantified in terms of the number of adherent thrombocytes per square $\mathrm{mm}$ and the thrombocyte covered area in a static assay. The thrombocyte covered area was related to the activation state of the thrombocytes.

\section{Experimental}

\subsection{Materials}

All solvents and low molecular weight substances were purchased from Merck KGaA (Darmstadt, Germany), Sigma (Steinheim, Germany) or Sigma-Aldrich (Munich, Germany) and applied as received, unless otherwise mentioned. PBS buffer was purchased from Sigma Aldrich $\left(8.0 \mathrm{~g} \mathrm{~L}^{-1} \mathrm{NaCl}, 1.15 \mathrm{~g} \mathrm{~L}^{-1}\right.$ $\mathrm{NaHPO}_{4}, 0.2 \mathrm{~g} \mathrm{~L}^{-1} \mathrm{KCl}$ and $0.2 \mathrm{~g} \mathrm{~L}^{-1} \mathrm{KH}_{2} \mathrm{PO}_{4}, \mathrm{pH} 7.4 ; \mathrm{GIBCO}^{\circledR}$ Cell Therapy Systems DPBS CTS ${ }^{\mathrm{TM}}$, Dulbecco's, AppliChem, Darmstadt, Germany) and the solution was used as received. Dialysis was performed in regenerated cellulose tubes from Spectrum laboratories (Spectra/Por ${ }^{\circledR} 6$ Dialysis membrane, molecular weight cut-off (MWCO) $1000 \mathrm{~g} \mathrm{~mol}^{-1}$, purchased from Roth, Karlsruhe, Germany).

IR spectra were recorded on a Nicolet Avatar 32 FT-IR with a Smart iTR accessory. Molecular weights were determined using a GPC (Agilent 1100 Series) including a UV detector (254 nm) as well as a refractive-index detector in comparison to pullulane standards.

hOG and hPG were synthesized by one-step anionic ringopening polymerization starting from 1,1,1-tris(hydroxymethyl)propane partially deprotonated $(10 \mathrm{~mol} \%)$ with potassium methylate solution (3.7 $\mathrm{M}$ in methanol) and glycidol to give compounds with a molecular weight of $2 \mathrm{kDa}$ (hOG; $M_{\mathrm{n}}=2.0$ $\left.\mathrm{kDa}, M_{\mathrm{w}}=3.1 \mathrm{kDa}\right)$ or $5 \mathrm{kDa}\left(\mathrm{hPG} ; M_{\mathrm{n}}=4.8 \mathrm{kDa}, M_{\mathrm{w}}=8.3 \mathrm{kDa}\right)$. Then, the hyperbranched glycerols were reacted with mesylchloride ( $5 \mathrm{~mol} \%$ ) in pyridine, washed, dried, and dialyzed to quantitatively yield a brown, honey-like substance. Mesylated hOG or hPG was dissolved in DMF and reacted with sodium azide ( 5 eq.) to give oligo- or polyglycerylazide. For the synthesis of the methylated compounds, the oligo- or polyglycerolazide was reacted with methyl iodide, yielding degrees of methylation of $61 \mathrm{~mol} \%$ (hOG-OMe) and $88 \mathrm{~mol} \%$ (hPG-OMe). The methylation leads to a calculated increase of the molecular weight to $2.2 \mathrm{kDa}$ (hOG-OMe) and 5.4 kDa (hPG-OMe). Catalytic hydrogenation of the azide groups of the hydroxy- or methoxy oligoand polyglycerols with $\mathrm{Pd} / \mathrm{C}$ gave the corresponding amine functionalized polyglycerols.

Linear, side chain methylated, mono amino terminated oligoglycerol (OG-OMe) was synthesized by anionic polymerization. As an initiator potassium-tert-butoxide was reacted at $90{ }^{\circ} \mathrm{C}$ with $N, N$-dibenzyl-3-aminopropan-1-ol in dry dimethoxyethane the formed tert-butanol and solvent were distilled off. The glycidyl methyl ether was dissolved in dimethoxyethane, added to the initiator and polymerized under an argon atmosphere at $90{ }^{\circ} \mathrm{C}$ overnight. Polymerization was quenched by the addition of water. After removal of the solvent, the polymer was precipitated in diethyl ether. Finally, the benzyl groups of $N, N$ dibenzyl-3-aminopropan-1-ol were hydrogenated to give the amino function by treatment with $10 \mathrm{wt} \% \mathrm{Pd} / \mathrm{C}$ in methanol at 5 bar hydrogen pressure for $48 \mathrm{~h}$. The crude product was filtered through Celite to give yellow-orange oil.

A detailed characterization of hOG, hPG, and OG-OMe can be found in the ESI. $\dagger$

\subsection{Membrane fabrication and functionalization}

Poly(ether imide) (PEI) (Ultem ${ }^{\circledR}$ 1000; GE-Plastics, Fairfield, USA; $M_{\mathrm{w}}=30000 \pm 10000 \mathrm{~g} \mathrm{~mol}-1, M_{\mathrm{n}}=12000 \pm$ $4000 \mathrm{~g} \mathrm{~mol}^{-1}$ ) flat sheet membranes were fabricated by continuous non-solvent induced phase separation. The PEI solution (17.5 wt\% PEI in $30 \mathrm{wt} \% \gamma$-butyrolactone and $52.5 \mathrm{wt} \%$ dimethylacetamide) was cast onto an endless steel band, immersed into deionized water at room temperature, thoroughly washed with deionized water, tempered $\left(10 \mathrm{~min}, 90{ }^{\circ} \mathrm{C}\right.$, Millipore water) and dried for storage.

The surface functionalization of the PEI membranes was carried out with $2 \mathrm{wt} \%$ aq. solutions of hOG, hPG, or OG-OMe $(\mathrm{pH}=11$ adjusted $)$ at $70{ }^{\circ} \mathrm{C}$ for $17 \mathrm{~h}$. Afterwards, the membrane 
was washed with deionized water. Furthermore, membranes treated under the same conditions at $\mathrm{pH} 11$ without adding glycerols were fabricated (PEI-pH 11).

Samples were stored in sodium azide solution (0.02 wt $\%$ $\mathrm{NaN}_{3}$ in Ampuwa ${ }^{\circledR}$ Spüllösung, Fresenius-Kabi, Bad Homburg, Germany) and were washed with Millipore water or, for biological testing, with Ampuwa ${ }^{\circledR}$ water before characterization.

\subsection{Physico-chemical characterization}

2.3.1 Small angle X-ray scattering (SAXS) measurements. SAXS measurements were performed with a Kratky-type instrument (SAXSess from Anton Paar, Austria). The scattered intensities were recorded with a cooled $\left(-40{ }^{\circ} \mathrm{C}\right) \mathrm{CCD}$ camera (PI-SCX, Princeton Instruments, USA) in the line collimation geometry and integrated into the one-dimensional scattering function $I(q)$ using SAXSQuant 1D software (Anton-Paar, Austria). Aqueous solutions of the polymers with concentrations in the range of 1.0 to $2.0 \mathrm{~g} \mathrm{~L}^{-1}$ were poured into vacuum-tight thin quartz capillaries at room temperature and placed in a TCS 120 temperature-controlled sample holder. The temperature was held constant at $20^{\circ} \mathrm{C}$. Background contributions from capillary and solvent scattering were subtracted from the sample scattering. The scattering vector $q$, defined as $q=4 \pi / \lambda \sin \theta$ where $\theta$ is the scattering angle and $\lambda$ the wavelength of the radiation, varied in the 0.1 to $6.0 \mathrm{~nm}^{-1}$ range.

Theoretical descriptions of the interpretation of the SAXS measurements are given in the ESI. $\dagger$

Samples of all membranes were analyzed by FT-ATR-IR spectroscopy (MagnaIR ${ }^{\mathrm{TM}}$ Spectrometer 550, Nicolet, Thermo Fisher Scientific, Waltham, MA, USA). The spectra were normalized to the signal of the $\mathrm{C}=\mathrm{O}$ stretching vibration at $1720 \mathrm{~cm}^{-1}$.

Functionalized and non-functionalized membrane samples were analyzed by X-ray induced photoelectron spectroscopy (XPS) (15 kV X-ray gun, monochromatic Al $\mathrm{K}_{\alpha}$ radiation, pass energy for region scan $20 \mathrm{eV}$, charge compensator, Kratos Axis Ultra DLD, Kratos-Analytical-Ltd., Manchester, United Kingdom). The photoelectrons were detected at different exit angles of $0^{\circ}, 45^{\circ}$ and $60^{\circ}$. All spectra were analyzed using software CasaXPS'т (Version 2.3.14, Casa Software Ltd., Teignmouth, United Kingdom), applying a single Shirley-Gauss algorithm to smoothen the signals if necessary and using Gauss shaped integrals to fit the signal shape for carbon (C1s) (C-C/C$\mathrm{H} / \mathrm{C}=\mathrm{C} 284.6 \mathrm{eV}$; C-N/C-O $286.2 \mathrm{eV}$; amide $287-288 \mathrm{eV}$; imide $288.3 \mathrm{eV}$; carboxylic acid $298 \mathrm{eV} ; \pi \rightarrow \pi^{*}$ shake-up $291.2 \mathrm{eV}$; additional signals if necessary) and oxygen (O1s) $(\mathrm{O}=\mathrm{C} 531.7$ $\mathrm{eV}, \mathrm{O}-\mathrm{C} 533.2 \mathrm{eV}$; amide 534-535 eV; $\pi \rightarrow \pi^{*}$ shake-up $538 \mathrm{eV}$; and additional signals).

2.3.2 Contact angle measurements. Contact angle measurements were performed with a DSA 100 (KrüssOptronic GmbH, Hamburg, Germany) using the captive bubble method in a water-air system ( 3 positions per sample, $n=26-30$ values in total per sample).

The roughness of the native and the functionalized PEI membranes was characterized by atomic force microscopy (AFM, tapping mode, NanoScope, Veeco, Mannheim, Germany) in PBS buffer. Surface areas of 1,10 , and $50 \mu \mathrm{m}^{2}$ were investigated for all membranes. Three different areas from three different samples were analyzed for each functionalization to predict the roughness homogeneity. Values for $R_{\mathrm{a}}$ (arithmetic average of absolute roughness values) and $R_{\mathrm{q}}$ (root mean squared roughness) in $\mathrm{nm}$ were calculated.

2.3.3 Tangential streaming potential. Tangential streaming potential measurements were performed using the Microslit Electrokinetic Setup (MES, ZetaSCIENCE GmbH, Dresden, Germany), equipped with a flat plate measuring cell. ${ }^{21}$ Measurements were carried out at $22 \pm 2{ }^{\circ} \mathrm{C}$ with aqueous 0.001 $\mathrm{M} \mathrm{KCl}$ solutions. After insertion into the cell body, the equilibration was completed with freshly prepared $0.001 \mathrm{M} \mathrm{KCl}$ solution until constant $\mathrm{pH}$ and conductivity were observed. Afterwards, the $\mathrm{pH}$ was changed stepwise in about 0.1 units (3 $\mathrm{pH}$ runs from $\sim 8 \rightarrow 3 \rightarrow 9 \rightarrow 3$ ) by addition of $0.1 \mathrm{M} \mathrm{KOH}$ or $\mathrm{HCl}$, respectively. The zeta potential $\zeta$ was calculated from streaming current $\mathrm{d} I / \mathrm{d} p$ data $\left(\zeta_{\mathrm{I}}\right)$. The calculations are based on a linear regression of data sampled at 5 pressure steps in each flow direction between 60 and 120 mbar along the channel.

\subsection{Biological characterization}

2.4.1 Protein adsorption. The protein adsorption was determined by incubating the membrane discs (28 $\mathrm{mm}, n=3$ ) in $5 \mathrm{~mL}$ protein solution [400 $\mu \mathrm{g}$ protein per $\mathrm{mL}$ ] in PBS buffer, pH 7.4, bovine serum albumin (BSA, Fraction V; Merck KGaA, Darmstadt, Germany), fibrinogen (Fraction I, Type I-S: bovine; Sigma-Aldrich, Munich, Germany), or lysozyme (human, $>100000 \mathrm{U} \mathrm{mg}^{-1}$, Sigma-Aldrich, Munich, Germany) for $16 \mathrm{~h}$ at room temperature under mild shaking. The protein concentration of the supernatant was determined colorimetrically (Tecan SPECTRA Fluor Plus, Männedorf, Switzerland) with the BCA protein assay (Thermo Fischer Scientific Inc., Waltham, MA, USA) with a detection limit of $20 \mu \mathrm{g} \mathrm{mL} \mathrm{m}^{-1}$.

Immunochemical labeling of surface adsorbed albumin and fibrinogen (mouse monoclonal anti-human albumin antibody labeled with DyLight 488 and goat polyclonal anti-human fibrinogen antibody labeled with $\mathrm{Cy} 3$, Dianova $\mathrm{GmbH}$, Hamburg, Germany) was performed after sample discs $(\varnothing=13$ $\mathrm{mm})$ were treated with human thrombocyte rich plasma $(1 \mathrm{~h}$ in a humidified atmosphere, 5 vol\% $\mathrm{CO}_{2}$ ). Paraformaldehyde fixed ( $4 \mathrm{wt} \%$ in phosphate buffered saline) and stained samples were processed with a confocal laser scanning microscope (LSM 510 Meta, Carl Zeiss MicroImaging GmbH, Jena, Germany; 100 fold primary magnification).

2.4.2 Thrombocyte adhesion and activation. According to the criteria of the Nordkem-workshop and the guidelines of the British Committee for standards in Haematology (BCSH) and International Society on Thrombosis and Haemostasis (ISTH), ${ }^{22,23}$ blood was obtained from apparently healthy subjects, who did not take any thrombocyte function inhibitors or other pharmaceuticals for at least 10 days. The study has been designed according to the current guidelines of the British committee for standards in hematology ${ }^{24}$ and was evaluated and approved by the institutional review committee before the start of the study. Hemogram values (e.g. numbers of red- and 
white-blood cells, hemoglobin, and hematocrit) were determined to be within the reference ranges. An adequate thrombocyte function was proven by assessing the reactivity of sodium citrate anticoagulated thrombocytes on equine type I collagen and epinephrine bitartrate as well as equine type I collagen and adenosine-5'-diphosphate (ADP) with the Dade ${ }^{\circledR}$ PFA-100 thrombocyte function analyzer (Siemens Healthcare Diagnostics, Marburg, Germany). ${ }^{22,25}$

Static thrombocyte adhesion tests were carried out in a 24 well plate format with $1 \mathrm{~mL}$ of adjusted thrombocyte rich plasma $\left(5 \times 10^{4}\right.$ thrombocytes per $\left.\mu \mathrm{L}\right)$ per sample $(\varnothing=13 \mathrm{~mm}$, $n=3$ per donor, six donors, incubated for $1 \mathrm{~h}$ in a humidified atmosphere, $\left.5 \mathrm{vol} \% \mathrm{CO}_{2}\right)$. Glutaraldehyde ( $1 \mathrm{wt} \%$ in Millipore water) was used to fix and visualize the adherent thrombocytes (glutaraldehyde induced fluorescence technique, GIFT). ${ }^{26}$ All samples were examined at the same predefined position using a confocal laser scanning microscope (LSM 510 Meta, Carl Zeiss MicroImaging $\mathrm{GmbH}$, Jena, Germany; 100 fold primary magnification). Numbers and areas of adherent thrombocytes were determined by an image-based automated process and averaged for each donor. ${ }^{27}$ Reported values display the arithmetic mean of adherent thrombocytes or covered areas from six analyzed donors (standardized by adjusting the number of adherent thrombocytes per microscopic field of view to $\mathrm{mm}^{2}$, irrespective of material roughness or topography). Medical grade silicone (Bess Medizintechnik GmbH, Berlin, Germany) was used as a negative control to monitor the physical functions of the platelets.

Gaussian distributions were tested using Kolmogorov and Smirnov tests. For repeated measures one-way ANOVA tests were performed considering $p$-values less than 0.05 as significant (Tukey adjusted).

\section{Results and discussion}

The aminated hOG and hPG (Fig. 1a) were synthesized by first establishing the hyperbranched oligo- or polyglycerol structure, ${ }^{28}$ followed by partial mesylation ( $5 \mathrm{~mol} \%$ of the hydroxyl groups), subsequent conversion of the mesyl groups to azide groups, and finally reduction to the free amines. ${ }^{29}$ The last step is quantitative as determined by IR spectroscopy. The methylated compounds were obtained by treating the oligo- or polyglycerolazides with methyl iodide prior to the reduction step. The degree of functionalization was determined by ${ }^{1} \mathrm{H}$ NMR spectroscopy and was $61 \mathrm{~mol} \%$ for hOG-OMe and $88 \mathrm{~mol} \%$ for hPG-OMe. The solution structures of the hOG and hPG can have an important impact on their diffusibility, influencing the surface functionalization kinetics. Therefore, the solution structures were investigated in mixtures of water (as good solvent) and acetone (as non-solvent) with ratios of $1: 0,1: 1$, and $1: 3$ with SAXS in the nanoscale range of $\pi / q_{\max }=0.5 \mathrm{~nm}$ to $\pi / q_{\min }=35 \mathrm{~nm}$. The SAXS patterns of hOG-OH, hPG-OH, and hPG-OMe are typical for the scattering of branched polymers shown as solid lines in ESI Fig. S2, $\dagger$ in which the data are in good agreement with the model. The radii of gyration decrease with an increasing amount of acetone from $1.31 \mathrm{~nm}$ to $1.10 \mathrm{~nm}$ for hOG-OH and from $1.79 \mathrm{~nm}$ to $1.49 \mathrm{~nm}$ for hPG-OH. This
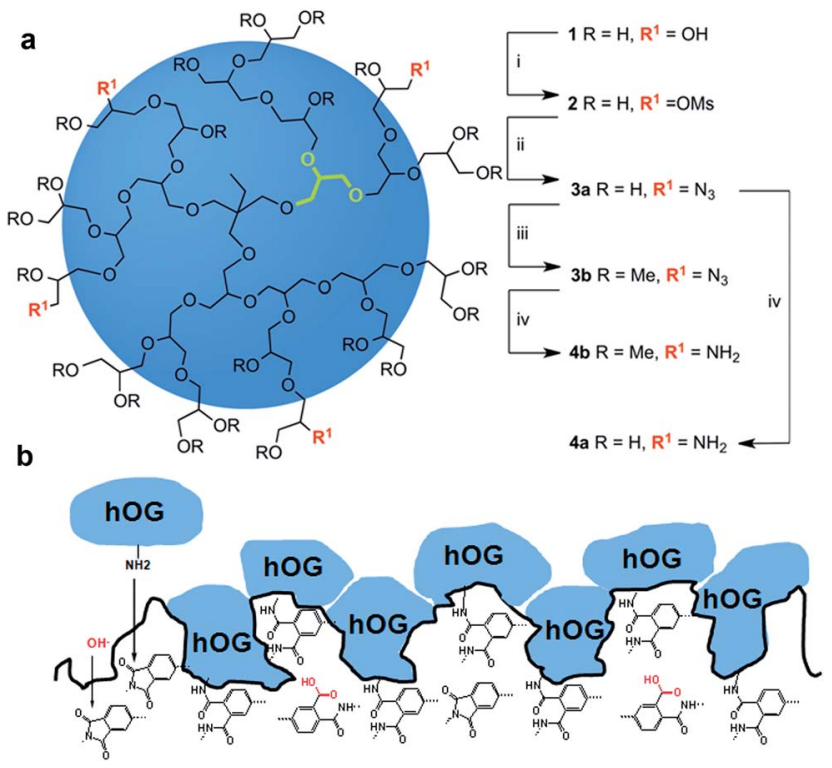

Fig. 1 (a) Chemical structure of the hyperbranched oligo- and polyglycerols and their synthetic conversion to the aminated, hydroxy or methoxy terminated polyethers used for surface functionalization. (i) $\mathrm{MsCl}\left(5 \mathrm{~mol} \%\right.$ ), pyridine, rt, $24 \mathrm{~h}$; (ii) $\mathrm{NaN}_{3}$ (5 eq.), DMF, $60^{\circ} \mathrm{C}, 3$ days; (iii) $\mathrm{KOH}, \mathrm{DMSO}, \mathrm{Mel}, \mathrm{rt}, 24 \mathrm{~h}$; (iv) $\mathrm{Pd} / \mathrm{C}, \mathrm{MeOH}, \mathrm{rt}, 3$ days. The structure represents an idealized segment of hOG/hPG with its partially functionalized end groups. In this work, two polymers with an average of 30 (hOG) and 75 (hPG) repeat units as multifunctional core polymers were used. (b) Schematic representation of a rough, hOG functionalized PEI surface, detailing the sites of attachment (amide linkages) as well as the formed free carboxylic acid groups at the interface of PEI and hOG. hPG attaches analogously. The polydispersity of hOG/hPG and the simultaneous attachment of each hOG/hPG molecule at several sites are intended to allow full surface coverage. On the left hand side, the linking reaction and the imide hydrolysis are exemplarily shown.

corresponds to a decrease of the excluded volume parameter from 0.58 to 0.37 for hOG-OH and from 0.65 to 0.37 for hPG$\mathrm{OH}$. These decreases indicate a change from a solution structure of a polymer in a good solvent to a largely collapsed polymer chain. In contrast, the radius of gyration in the case of hPG-OMe decreased from $11.83 \mathrm{~nm}$ to $3.62 \mathrm{~nm}$. These values and the large extent of the reduction of $R_{\mathrm{g}}$ are much too large for single polymer chains with a molar mass of $5000 \mathrm{~g} \mathrm{~mol}^{-1}$, and can be explained if clusters of $c a .5$ to 10 polymer chains are formed in water. This is in accordance with the excluded volume parameter decreasing from 0.89 to 0.75 . Such high values close to 1 are characteristic of polymers in stretched conformations, which can here be tentatively explained by a structure that resembles an expanded string-of-pearls.

\subsection{Surface functionalization}

In the next step, different hOG and hPG were used for surface functionalization of PEI membranes, and the effectivity of the reaction was investigated by studying the membrane surface before and after functionalization in the dry (FT-IR, XPS) as well as the wet state (contact angle, streaming potential measurements). The surface functionalization was performed at $\mathrm{pH} 11$ 
and $70{ }^{\circ} \mathrm{C}$, as these conditions were shown to maximize the functionalization reaction without leading to changes in the surface structure in earlier experiments. ${ }^{30}$ The PEI membranes were produced by continuous non-solvent induced phase separation. In this way, a free standing, non-woven free membrane was produced. In SEM, the produced membrane showed an asymmetric morphology, with small pores $<10 \mathrm{~nm}$ on the upper side and larger pores $>500 \mathrm{~nm}$ on the bottom side (ESI Fig. S1 $\dagger$ ). The surface roughness of the upper side was determined by AFM (ESI Table S1 $\dagger$ ). Only the upper side of the membrane was used in the further experiments for characterizing the surface functionalization and thrombocyte adhesion. The membranes did not contain extractable solvent residues as shown by GC analyses. A schematical view of the surface functionalization is shown in Fig. 1b. The surface functionalization step with hOG did not change the membrane morphology (see ESI Fig. S1†) or surface roughness (see ESI Table S1†).

The surface functionalization was investigated by spectroscopic methods proving the presence of hOG and hPG, respectively. In FT-ATR-IR spectroscopy (Fig. 2) additional peaks were observed at 1000-1200 $\mathrm{cm}^{-1}$ (C-O-C stretch), 1500$1700 \mathrm{~cm}^{-1}$ (amide I and II) and $2800-3800 \mathrm{~cm}^{-1}$ (C-H stretch of $\mathrm{CH}_{2}$-groups) after functionalization. Furthermore, spectra of the hydroxyl terminated hOG/hPG showed an additional IR vibration band at $3100-3800 \mathrm{~cm}^{-1}$, but no different intensities were observed for the other bands compared to the spectrum of methylated compounds with the same molecular weight. hPG showed increased signals compared to hOG at the wavenumbers corresponding to the ethylene glycol units (1000-1200 and 2800-3100 $\mathrm{cm}^{-1}$ ), suggesting a thicker layer of hPG than of hOG. In comparison to the hyperbranched structures, the linear OG-OMe functionalized PEI membranes showed the introduction of the oligoglycerol by similar IR vibration bands, as above, due to the similar structure, but with a reduced intensity of the signals. This indicates a higher grafting density of the hyperbranched structures than of the linear structures, which could be related to the molecular structure enabling multivalent binding. The multivalent binding which occurred is supported by a degradation experiment, in which crosslinked structures remained after functionalization with the hyperbranched glycerols, while full dissolution was observed after functionalization of PEI with linear structures.

XPS analyses of the hOG, hPG, or OG-OMe functionalized PEI membranes were performed as a surface sensitive method giving additional information on the covalent attachment. In accordance with earlier experiments, ${ }^{31}$ the angle between the detector and the surface was varied from $0^{\circ}$ to $75^{\circ}$ to increase the number of photoelectrons related to the surface of the sample and therefore to enable the analysis of the introduced thin surface layers. A change of the quantitative elemental composition confirmed the surface functionalization of the PEI membranes with hOG/hPG. The oxygen content was increased, while a reduction of the carbon content was detected (ESI, Table $\mathrm{S} 2 \dagger)$. Interestingly, no clear alteration between the different investigated detector angles was observed. In the case of the methoxylated compounds, the measured elemental composition corresponds to the calculated composition of hOG/hPG, while for hydroxy terminated hOG/hPG the determined values were between the calculated composition of the hyperbranched structure and PEI (see ESI for all values $\dagger$ ).

The surface coverage could be verified by the analyses of the chemical composition in the XPS region scans of the functionalized PEI membranes (ESI, Table S3†). Here, the $\mathrm{C}-\mathrm{O} / \mathrm{C}-\mathrm{N}$ signals at approximately $286.4 \mathrm{eV}$ were strongly increased compared to pure PEI-0 and alkaline treated PEI-pH 11. These observations were in accordance with the finding of the strongly increased ratio of $\mathrm{O}-\mathrm{C}$ bonds $(\sim 533.2 \mathrm{eV})$ in the $\mathrm{C} 1 \mathrm{~s}$ region scans. The covalent binding of the oligo- and polyethers could be observed in the appearing amide functions in the recorded C1s and O1s spectra. Furthermore, hOG-OMe and hPG-OMe showed an additionally occurring chemical function in the $\mathrm{O} 1 \mathrm{~s}(\sim 533 \mathrm{eV})$ and partially in the C1s $(285 \mathrm{eV})$ region scan spectra of the XPS being related to the methoxy end groups, which are present in high quantities. The nitrogen signals could not be individually analyzed because of the low signal to noise ratio.

Comparing the dry state physicochemical analyses, the results from the XPS analysis show the surface functionalization reaction to be more effective for methylated hyperbranched structures than for the hydroxy terminated compounds (XPS).
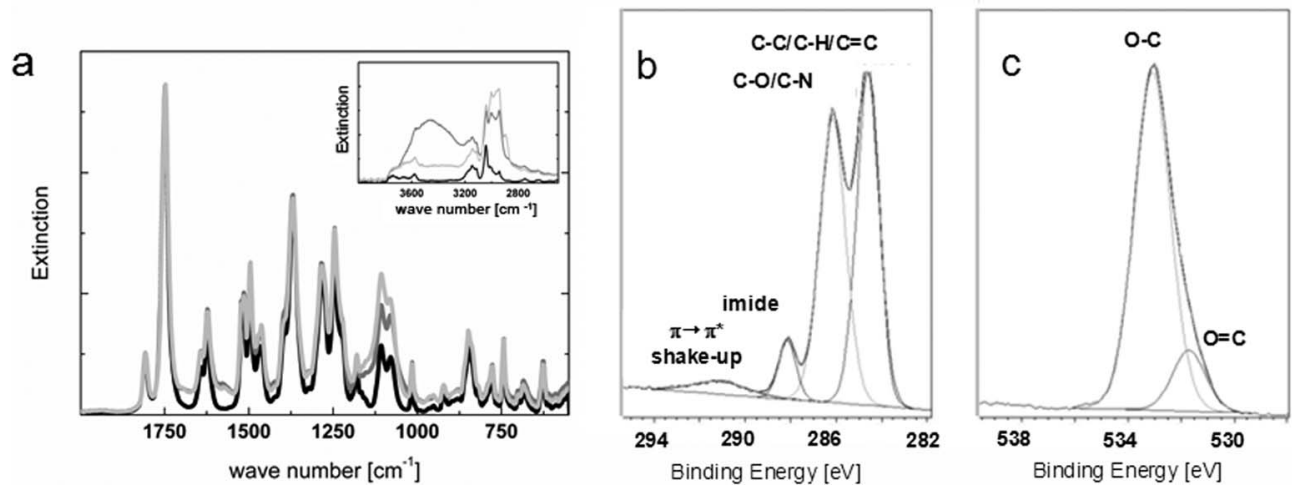

Fig. 2 (a) Comparison of FT-ATR-IR spectra of untreated PEI-O (black), hPG-OH functionalized (gray), and hPG-OMe (light gray). Representative XPS spectra of the $\mathrm{Cls}$ (b) and O1s (c) region scan in the XPS for the PEI membrane functionalized with hOG-OH, indicating a decrease in the carbon content and an increase in the oxygen content compared to unfunctionalized PEI. 
FT-IR analysis proves a higher weight amount of hPG being bound on the surface than of the smaller hOG.

\subsection{Properties of surface in contact with the aqueous phase}

While proving the presence of hOG and hPG on the PEI surface as well as the covalent attachment, the methods measured on dry samples provide no insight into the behavior of the functionalized surfaces under application relevant conditions. Therefore, characterization of wet samples was performed. It was observed that the contact angles varied notably between the untreated, alkaline treated, and surface functionalized membranes (Table 1). The hOG or hPG functionalized surfaces had all $\Theta_{\mathrm{adv}}$ values below the Berg limit of $60^{\circ}$ and are therefore representing hydrophilic surfaces with low unspecific protein binding capacities, ${ }^{32}$ with hPG-OH giving the lowest $\Theta_{\mathrm{adv}}$ value of this group. This observation is likely to be related to good surface shielding, but is also in line with the observation that functionalization with oligomers bearing free hydroxyl end groups showed lower contact angles than methoxy terminated hOG/hPG. ${ }^{33}$ This tendency was enhanced for hPG compared to hOG, which can be attributed to the increased amount of terminal hydroxyl functions introduced to the surface. The linear side chain methylated analogue OGOMe included in this study showed a similar contact angle to the methylated hOG and hPG in this analysis. Untreated PEI-0 had the highest contact angles $\left(\Theta_{\mathrm{adv}}=87^{\circ}\right)$, while the PEI-pH 11 membranes showed the lowest contact angles $\left(\Theta_{\mathrm{adv}}=22^{\circ}\right)$, which can be related to the large number of freely accessible carboxylic acid groups on the membrane.

Zeta potential measurements were conducted to study the properties of the interface between the bulk material and solution. In the case of PEI-0 and PEI-pH 11, hydroxide ion adsorption/desorption processes (shown by a continuous decrease of the $\zeta$ potential with increasing $\mathrm{pH}$ ), deprotonation of carboxylic acids (evidenced by a plateau with constant $\zeta$ in the graph), and

Table 1 Contact angle and zeta potential measurements for untreated PEI-0, alkaline treated PEI-pH 11, hOG/hPG functionalized PEI membranes compared to data obtained for PEI functionalized with OG-OMe

\begin{tabular}{|c|c|c|c|c|c|}
\hline \multirow[b]{2}{*}{$\begin{array}{l}\text { Sample } \\
\text { ID }^{a}\end{array}$} & \multicolumn{3}{|c|}{ Contact angle in water-air } & \multicolumn{2}{|c|}{ Zeta potential } \\
\hline & $\begin{array}{l}\Theta_{\mathrm{adv}} \\
{\left[{ }^{\circ}\right]}\end{array}$ & $\begin{array}{l}\Theta_{\text {rec }} \\
{\left[{ }^{\circ}\right]}\end{array}$ & $\begin{array}{l}\text { Hysteresis } \\
{\left[^{\circ}\right]}\end{array}$ & $\begin{array}{l}\mathrm{pH} \text { at IEP } \\
\left(\zeta_{\mathrm{I}}=0\right)\end{array}$ & $\begin{array}{l}\zeta_{\mathrm{I}} \text { at } \mathrm{pH} \\
7.4[\mathrm{mV}]\end{array}$ \\
\hline PEI-0 & $87 \pm 4.5$ & $42.6 \pm 4.0$ & 44.4 & 2.8 & -17 \\
\hline PEI-pH 11 & $22.4 \pm 1.3$ & $25.4 \pm 3.6$ & -3.0 & 2.6 & -33 \\
\hline hOG-OH & $50.0 \pm 3.0$ & $25.9 \pm 2.8$ & 24.1 & 5.3 & -24 \\
\hline hOG-OMe & $57.9 \pm 1.7$ & $39.3 \pm 2.8$ & 18.6 & 6.2 & -13 \\
\hline hPG-OH & $31.2 \pm 2.1$ & $27.0 \pm 4.6$ & 4.2 & 5.6 & -16 \\
\hline hPG-OMe & $59.7 \pm 1.4$ & $35.2 \pm 4.3$ & 24.5 & 5.5 & -24 \\
\hline OG-OMe & $56.4 \pm 1.2$ & $33.7 \pm 5.0$ & 22.7 & 3.2 & -37 \\
\hline
\end{tabular}

${ }^{a}$ PEI-0: untreated PEI membrane; PEI-pH 11: pH 11 treated PEI membrane; hOG-OX: hyperbranched oligoglycerol functionalized PEI membranes, with hydroxyl $(\mathrm{X}=\mathrm{H})$ or methoxy $(\mathrm{X}=\mathrm{Me})$ end groups. hPG-OX: hyperbranched polyglycerol functionalized PEI membranes, with hydroxyl $(\mathrm{X}=\mathrm{H})$ or methoxy $(\mathrm{X}=\mathrm{Me})$ end groups (typical error for IEP $\leq \pm 0.1 ; \zeta$ for $20 \mathrm{mV} \leq|\zeta| \leq 40 \mathrm{mV} \leq \pm 2 \mathrm{mV}$ otherwise $5 \%$ ). imide ring cleavage reactions (identified by a minimum of the curve) were observed. These processes were also seen for hOGOMe, however, the plateau at $\zeta_{\mathrm{I}}$ values $>0$ is likely to be related to the (de)protonation of amino groups rather than carboxylic acids, corresponding to data received from aminated PEI surfaces. ${ }^{34}$ This corresponds to the higher isoelectric point (IEP) of this functionalized surface compared to the other hOG functionalized membranes and indicates that not all amino groups reacted with imide moieties in the functionalization with hOG-OMe. In contrast, the other hOG and hPG functionalized surfaces (exemplarily shown for hPG-OMe in Fig. 3) displayed a basically linear behavior of $\zeta_{\mathrm{I}}$ depending on the $\mathrm{pH}$, which correlates with the hydroxide adsorption/desorption as the potential determining mechanism. This can be rationalized by a complete surface shielding through the hyperbranched glycerols, leaving carboxylic acid and imide groups inaccessible (Fig. 3). This highlights that the strategy of multivalent attachment to yield full surface coverage was successful. Interestingly, the string-of-pearls solution structure of hPG-OMe did not negatively influence the surface functionalization.

Comparing the physicochemical analyses in the wet state, the surface shielding effect is similar for the hydroxy and methoxy terminated species, which contrasts the dry state investigation suggesting a larger amount of the methoxy terminated species being bound to the surface. However, similar to the dry state investigation, the surface shielding is more efficient with hPG than with hOG. A summary of all physicochemical analyses is given in Table 2 .

\subsection{Protein adsorption}

It was shown that compounds potentially interfering with the following experiments such as chemical residues or endotoxins

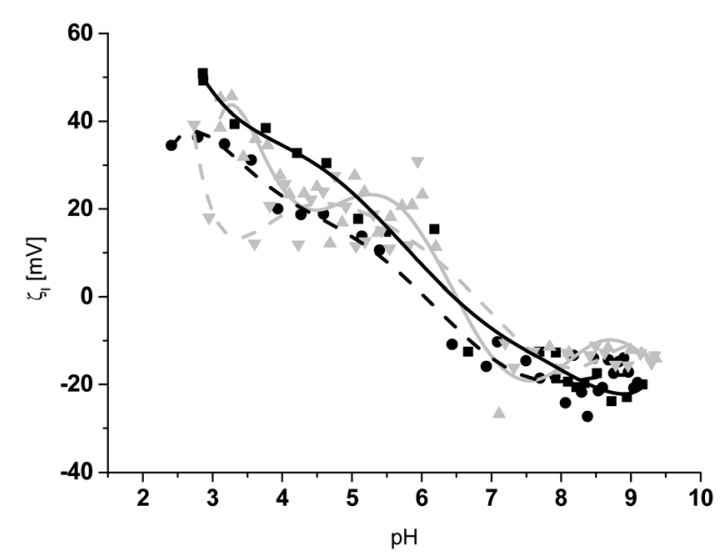

Fig. $3 \zeta_{1}$ plot of hOG-OMe $(\Delta:$ run $\mathrm{pH} 3 \rightarrow$ 9; $\mathbf{\nabla}$ : run pH $9 \rightarrow 3$ ) and hPG-OMe ( $\mathbf{\square}$ : run $\mathrm{pH} 3 \rightarrow$ 9; $\bullet$ : run $\mathrm{pH} 9 \rightarrow 3$ ). For hOG-OMe the plateau between $\mathrm{pH} 4$ and 6 at positive $\zeta_{1}$ values suggest free amino groups, while the pronounced minimum of the curve at $\mathrm{pH} 7.5$ indicates imide cleavage during the measurements, which can be interpreted by some parts of the PEI membrane surface being exposed during the measurements. In the case of hPG-OMe, which is representative also for the other hOG/hPG functionalized surfaces, mainly $\mathrm{OH}^{-}$adsorption was observed, which suggests a complete surface coverage. The lines (grey hOG-OH, black: hPG-OH, compact pH $3 \rightarrow$ 9, dashed $\mathrm{pH} 9 \rightarrow 3$ ) are added as guides to the eye. 
Table 2 Summary of the physicochemical characterization

\begin{tabular}{|c|c|c|c|c|c|c|}
\hline \multirow[b]{2}{*}{ Scientific question } & \multirow[b]{2}{*}{ Method } & \multicolumn{2}{|l|}{ hOG } & \multicolumn{2}{|l|}{ hPG } & \multirow[t]{2}{*}{ OG-OMe } \\
\hline & & $-\mathrm{OH}$ & -OMe & $-\mathrm{OH}$ & $-\mathrm{OMe}$ & \\
\hline Wt.-amount bound & IR & ++ & ++ & +++ & +++ & + \\
\hline Covalent attachment & XPS & + & + & + & + & + \\
\hline Surface shielding & $\zeta, \mathrm{CA}$ & + & + & ++ & + & $(+)$ \\
\hline
\end{tabular}

were not present, or that their influence could be verifiably excluded (for details see ESI $\dagger$ ).

Protein adsorption on the membranes was quantified for BSA, fibrinogen, and (for some samples) lysozyme using an indirect measurement with a BCA assay (Fig. 4). The hOG or hPG functionalized PEI membranes showed a reduced protein adsorption compared to PEI-0 and PEI-pH 11, but also compared to OG-OMe, as well as the previously studied PEGs and OEGs. ${ }^{12}$ The lowest amounts were observed for hPG-OH, with less than $3.4 \mu \mathrm{g} \mathrm{cm} \mathrm{cm}^{-2}$ of adsorbed mass for all tested proteins. Furthermore, there was no notable difference between the adsorption of different proteins on hOG or hPG functionalized surfaces. This negates a significant influence of molecular weight or end groups on the protein adsorption for these samples, while differences were observed for the non-functionalized PEI membranes and the OG-OMe functionalized membranes. Especially the positively charged lysozyme would adhere to surfaces with free carboxylic acid groups, most likely because of electrostatic interactions.

While the BCA-based method is fast and generally reliable, immunostaining techniques allow for a spatial analysis of the protein adsorption pattern. Therefore, after contact of the samples with thrombocyte-rich plasma, the samples were stained with fluorescently labelled human serum albumin antibodies and human fibrinogen antibodies. In the case of PEI0 and PEI-pH 11, albumin was adsorbed to the complete surface

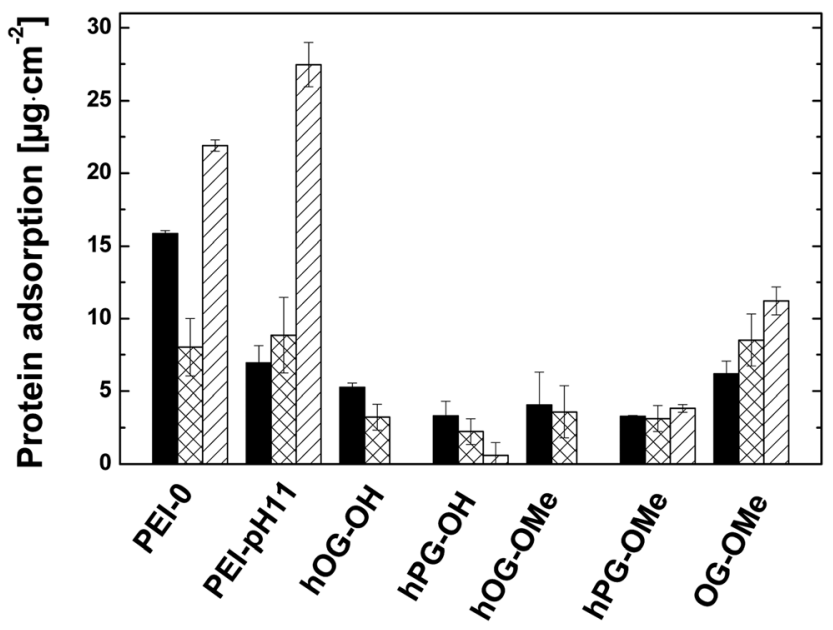

Fig. 4 Quantification of protein adsorption in an indirect BCA assay. (a) Fibrinogen (black), bovine serum albumin (checked), lysozyme (lined).
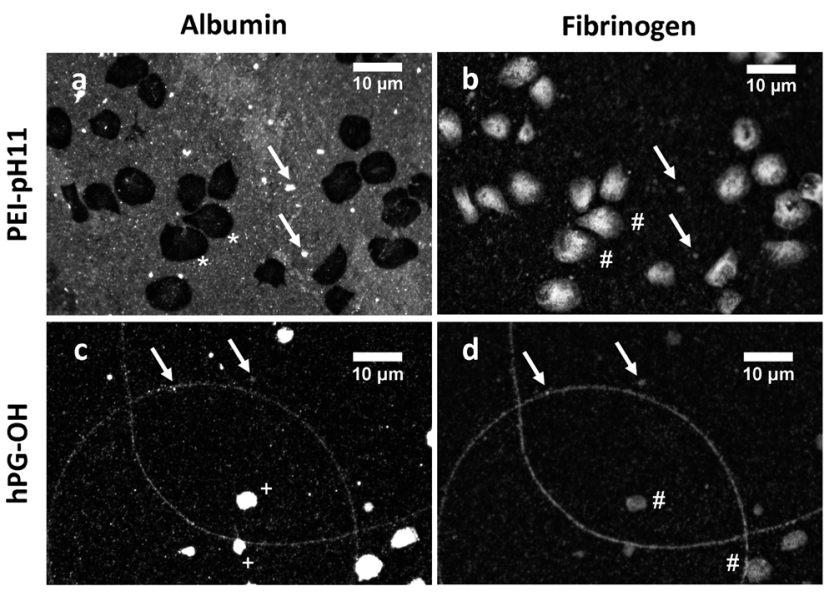

Fig. 5 Immunocytochemical staining of human albumin after treatment with thrombocyte rich plasma of untreated PEI-pH 11 (a) and $\mathrm{hPG}-\mathrm{OH}$ (c) and immunocytochemical staining of human fibrinogen in $\mathrm{PEI}-\mathrm{pH} 11$ (b) and hPG-OH (d). Due to the high staining degree of $\mathrm{PEI}-\mathrm{pH} 11$ (b) the thrombocytes can be seen. $\left({ }^{*}\right)$ Areas covered with adherent, fully spread thrombocytes, which do not show anti-albumin antibody binding. (+) Albumin covered thrombocytes in low stages of activation (early to intermediate pseudopodial). ${ }^{35,36}$ (\#) Fibrinogen covered thrombocytes (cell membrane bound plasma fibrinogen and/ or degranularized from $\alpha$-granula) in (b) strongly activated thrombocytes (fully spread) and (d) low stages of activation (early to intermediate pseudopodial). ${ }^{37}$ Arrows indicate areas with co-localized albumin and fibrinogen.

area, with some areas displaying larger agglomerates of albumin. The strong staining appears in Fig. 5a as a bright background. In the case of hPG-OH (Fig. 5c), albumin would only adsorb at some sites forming agglomerates, while large areas did not adsorb any albumin. This can be related to certain not well functionalized areas, e.g. pores. The hOG-OMe and hPG-OMe surface functionalized samples (but not hOG-OH), as well as OG-OMe, behaved in a manner similar to hPG-OH in immunostaining. A comparison of fibrinogen adsorption on the two samples shows a lower overall fibrinogen adsorption than for albumin, which is partially related to fibrinogen's lower concentration in blood than albumin, but also confirms the results from the BCA assay. Furthermore, a more homogeneous adsorption of fibrinogen was also found on PEI-0, while adhesion to hPG-OH was only observed at selected sites. The protein agglomerates of albumin and fibrinogen are co-localized, which supports the conclusion of presence of non-functionalized areas such as pores.

The staining behavior of the thrombocytes was enlightening. Thrombocytes are not stained by the anti-albumin antibody in PEI-pH 11, but they are strongly stained in hPG-OH. This observation correlates with the activation state of the thrombocytes, because non-activated thrombocytes are covered with albumin, while activated thrombocytes are not. The different activation states are further evidenced by the different spreading of the thrombocytes seen in the two pictures (Fig. 5). The lower intensity of the fibrinogen staining of the thrombocytes on hPG-OH compared to the albumin staining might be related to the fact that fibrinogen in non-activated 
thrombocytes is predominantly located internally, while albumin is localized on the cell surface. ${ }^{22,35}$ Therefore, the only slightly activated thrombocytes on hPG-OH are stained less intensively than the thrombocytes in PEI-pH 11, which are more activated.

Summarizing the protein adsorption assays, all hOG and hPG functionalized surfaces were similarly effective in reducing
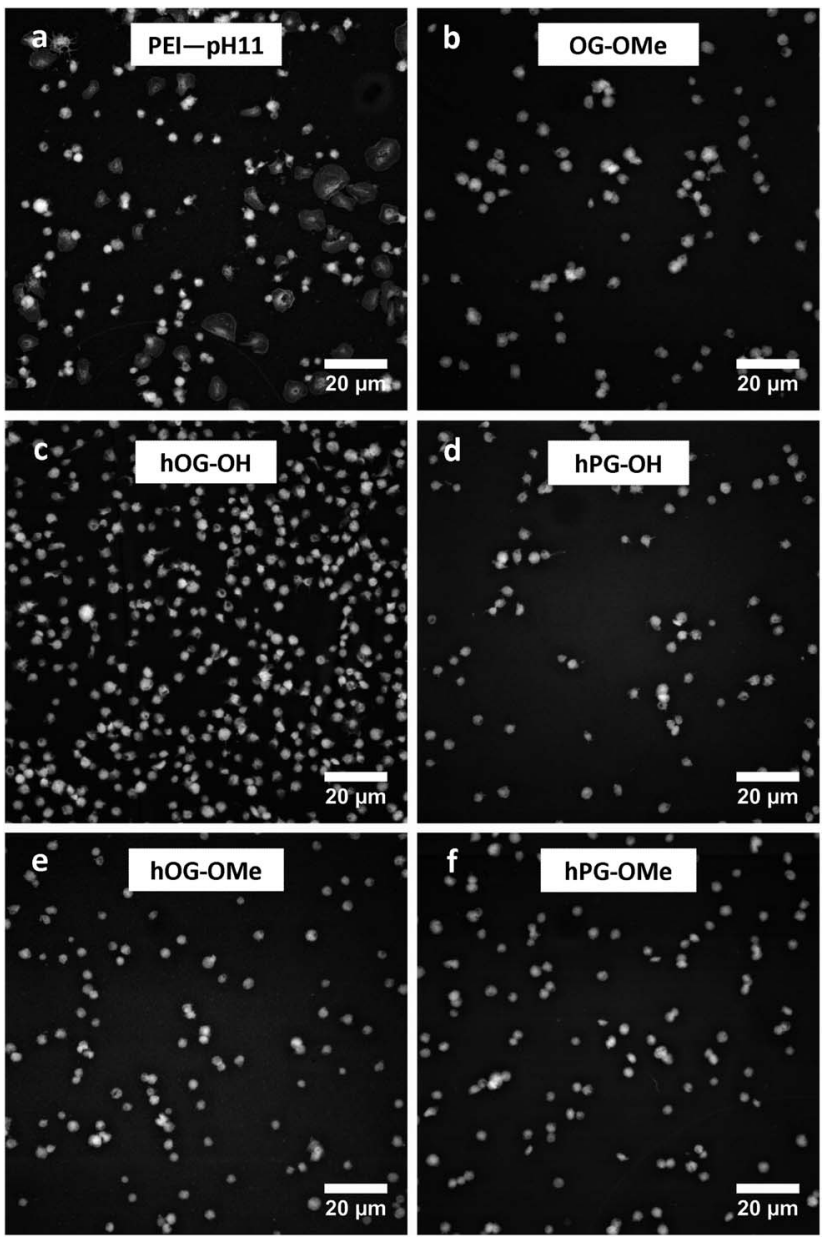

Fig. 6 Thrombocytes adhered to PEI-pH 11 and differently functionalized PEI surfaces. In PEI-pH 11 there is a significant number of activated thrombocytes visible, while the thrombocytes adhering to hOGor hPG-functionalized surfaces are not activated. protein adsorption, and performed better than PEI-0, PEI-pH 11, and OG-OMe. The functionalization performed here seems to be more effective in providing protein resistance than reported for poly(vinylidene fluoride) membranes functionalized with hyperbranched polyglycerols only attached at one site $(\gamma$-globulin attachment reduced to $25 \mathrm{wt} \%$ of the original value $^{38}$ or PEG brushes and network structures on poly(vinylidene fluoride) membranes (adhesion of BSA, fibrinogen, and $\gamma$-globulin reduced to $20 \mathrm{wt} \%){ }^{39}$

While protein adsorption in the literature is generally connected to lower cell adhesion, this is not necessarily the case, ${ }^{\mathbf{1 3}}$ and was investigated here by the detailed study of thrombocyte adhesion and activation. Representative confocal laser scanning microscopic images of adherent thrombocytes on PEI-pH 11 and all functionalized PEI membranes are depicted in Fig. 6. Adherent thrombocytes on all functionalized samples show predominantly low stages of activation (early to intermediate pseudopodial), in contrast, an increased number of strongly activated thrombocytes are present (fully spread) on the PEI-pH 11 membrane (100-fold primary magnification, GIFT staining). On PEI-0, similar results were found as on PEI-pH 11, with a tendency of more spread thrombocytes. The end groups as well as the molecular weight of hOG/hPG obviously did not influence the thrombocyte activation.

The quantification of adherent thrombocytes on the PEI surfaces (Fig. 7, left) shows similar numbers on all samples with the exception of hOG-OH. A non-complete surface shielding might be the reason here for this observation (see above). It can only be speculated why hOG-OH shows a much higher number of adhering thrombocytes than hOG-OMe, despite the methylation degree of only $61 \mathrm{~mol} \%$. One possible explanation might be an improved interaction of the methylated hyperbranched structures on the surface compared to the hydroxyl terminated compounds, resulting in better surface shielding, which is supported by the XPS data. Furthermore, while it was suggested that surfaces displaying - $\mathrm{OH}$ groups decrease protein adsorption, there are diverse reports on the influence of $-\mathrm{OH}$ groups on cell adhesion. ${ }^{\mathbf{4 0 , 4 1}}$ The smaller number of adhering thrombocytes on hPG-OH compared to hOG-OH could be interpreted due to differences in displaying the $-\mathrm{OH}$ groups and molecule dynamics, or by an altered tendency of the surfaces to induce conformational changes of adsorbed proteins, which might lead to the presentation of cryptic epitopes involved in cell
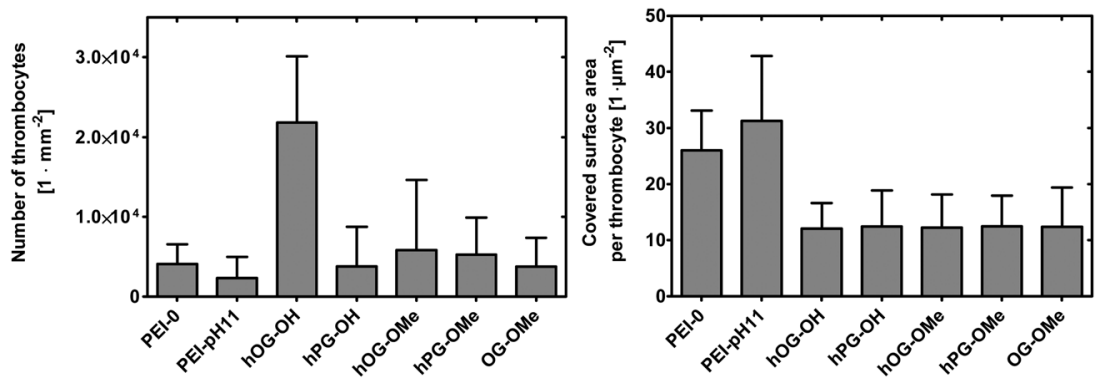

Fig. 7 Numbers of adherent thrombocytes (left) and covered areas per thrombocyte (right) of hOG/hPG-functionalized PEI membranes in comparison to PEI-0, alkaline treated PEI-pH 11 and glycerol functionalized PEI membranes, determined in the static thrombocyte adhesion experiment ( 6 donors, $n=3$ samples each, median \pm standard deviation). 
binding. ${ }^{42}$ Definite differences between the samples were observed when also evaluating the area covered per thrombocyte (Fig. 7, right). The glycerol functionalized surfaces (including hOG, hPG and OG-OME functionalizations) resulted in much smaller covered areas, corresponding to a low state of activation of the thrombocytes, compared to PEI-0 and PEI-pH 11. This is a remarkable result in comparison to the previously studied OEG and PEG functionalized PEI membranes, on which thrombocytes were fully spread and were in higher activation states. ${ }^{12}$ Generally, in biomedical applications, thrombocyte adhesion tends to be well tolerated if not accompanied by thrombocyte activation, and the low observed activation of adhering thrombocytes shown in the experiments is an important step in the direction of hemocompatible surfaces.

Furthermore, potential drawbacks that were reported for the application of OEG and PEG, ${ }^{\mathbf{1}}$ including instability at higher temperatures and oxidative conditions causing problems in fabrication and handling, degradation in vivo in the presence of enzymes, ${ }^{43}$ which in some cases led to the formation of toxic metabolites, and formation of PEG-binding antibodies resulting in immunological responses ${ }^{\mathbf{4 4}}$ were overcome by applying glycerol structures as reported here.

\section{Conclusions}

The physicochemical analysis showed that the strategy to increase surface coverage and shielding of polyethers that had been grafted to rough membranes by employing multivalent attachment of branched structures instead of linear molecules was successful, as shown for hOG and hPG. This is significant, as it contradicts the established structure-activity relationships gained with smooth model surfaces. Protein adsorption not only of albumin but also of fibrinogen could be decreased with this surface functionalization. Interestingly, here we could also demonstrate that the multivalent hPG-OH attachment strongly reduced the activation of adhering thrombocytes. This could be observed by analyzing the area covered per thrombocyte as well as the protein distribution pattern determined in immunostaining experiments, although the absolute number of adhering thrombocytes did not change much compared to the already not strongly thrombogenic non-functionalized PEI surface. As the only hydrolyzable bond of hPG is the slowly hydrolyzing amide bond to the polymer backbone and the polyglycerols are more resistant to oxidation than PEGs, it is anticipated that the surface functionalization is stable in vitro and in vivo for a prolonged time. The presented strategy is an important step on the way to functional materials with a hemocompatible surface showing low protein adsorption, low thrombocyte adhesion, and low thrombocyte activation as needed for applications of biomaterials intra- and extracorporeally.

\section{Acknowledgements}

This work was partially funded by the Federal Ministry of Education and Research (BMBF) through grant no. 1315696 (Poly4Bio BB).

\section{References}

1 K. Knop, R. Hoogenboom, D. Fischer and U. S. Schubert, Angew. Chem., Int. Ed., 2010, 49, 6288-6308.

2 C. Werner, M. F. Maitz and C. Sperling, J. Mater. Chem., 2007, 17, 3376-3384.

3 T. Satomi, Y. Nagasaki, H. Kobayashi, T. Tateishi, K. Kataoka and H. Otsuka, J. Nanosci. Nanotechnol., 2007, 7, 2394-2399.

4 J. Bluemmel, N. Perschmann, D. Aydin, J. Drinjakovic, T. Surrey, M. Lopez-Garcia, H. Kessler and J. P. Spatz, Biomaterials, 2007, 28, 4739-4747.

5 R. Fukai, P. H. R. Dakwa and W. Chen, J. Polym. Sci., Part A: Polym. Chem., 2004, 42, 5389-5400.

6 K.-M. Xiu, N.-N. Zhao, W.-T. Yang and F.-J. Xu, Acta Biomater., 2013, 9, 7439-7448.

7 H. Hu, X. B. Wang, S. L. Xu, W. T. Yang, F. J. Xu, J. Shen and C. Mao, J. Mater. Chem., 2012, 22, 15362-15369.

8 A. R. Statz, J. Kuang, A. E. Barron, I. Szleifer and P. B. Messersmith, Biointerphases, 2009, 4, FA22-FA32.

9 C. Sperling, M. F. Maitz, S. Talkenberger, M.-F. Gouzy, T. Groth and C. Werner, Biomaterials, 2007, 28, 3617-3625.

10 E. Ostuni, R. G. Chapman, R. E. Holmlin, S. Takayama and G. M. Whitesides, Langmuir, 2001, 17, 5605-5620.

11 D. J. Irvine, A. M. Mayes, S. K. Satija, J. G. Barker, S. J. SofiaAllgor and L. G. Griffith, J. Biomed. Mater. Res., 1998, 40, 498509.

12 A. T. Neffe, M. v. Ruesten-Lange, S. Braune, K. Luetzow, T. Roch, K. Richau, F. Jung and A. Lendlein, Macromol. Biosci., 2013, 1720-1729.

13 M. Lange, S. Braune, K. Luetzow, K. Richau, N. Scharnagl, M. Weinhart, A. T. Neffe, F. Jung, R. Haag and A. Lendlein, Macromol. Rapid Commun., 2012, 33, 1487-1492.

14 R. Du, B. Gao and Y. Li, Appl. Surf. Sci., 2013, 274, 288-294. 15 J. Pei, H. Hall and N. D. Spencer, Biomaterials, 2011, 32, 8968-8978.

16 A. Sunder, R. Mulhaupt, R. Haag and H. Frey, Adv. Mater., 2000, 12, 235-239.

17 R. K. Kainthan, S. R. Hester, E. Levin, D. V. Devine and D. E. Brooks, Biomaterials, 2007, 28, 4581-4590.

18 C. Siegers, M. Biesalski and R. Haag, Chem.-Eur. J., 2004, 10, 2831-2838.

19 M. Wyszogrodzka and R. Haag, Biomacromolecules, 2009, 10, 1043-1054.

20 M. Weinhart, T. Becherer, N. Schnurbusch, K. Schwibbert, H.-J. Kunte and R. Haag, Adv. Biomater., 2011, B501-B510.

21 R. A. van Wagenen and J. D. Andrade, J. Colloid Interface Sci., 1980, 76, 305-314.

22 F. Jung, S. Braune and A. Lendlein, Clin. Hemorheol. Microcirc., 2013, 53, 97-115.

23 S. Braune, M. Grunze, A. Straub and F. Jung, Biointerphases, 2013, 8, 33.

24 P. Harrison, I. Mackie, A. Mumford, C. Briggs, R. Liesner, M. Winter and S. Machin, Br. J. Haematol., 2011, 155, 30-44.

25 E. J. Favaloro, Semin. Thromb. Hemostasis, 2008, 34, 709-733.

26 R. D. Frank, H. Dresbach, H. Thelen and H.-G. Sieberth, J. Biomed. Mater. Res., 2000, 52, 374-381. 
27 S. Braune, G. Alagöz, B. Seifert, A. Lendlein and F. Jung, Clin. Hemorheol. Microcirc., 2012, 52, 349-355.

28 A. Sunder, R. Hanselmann, H. Frey and R. Muelhaupt, Macromolecules, 1999, 32, 4240-4246.

29 S. Roller, H. Zhou and R. Haag, Mol. Diversity, 2005, 9, 305316.

30 W. Albrecht, B. Seifert, T. Weigel, M. Schossig, A. Holländer, T. Groth and R. Hilke, Macromol. Chem. Phys., 2003, 204, 510-521.

31 M. v. Ruesten-Lange, K. Luetzow, A. T. Neffe and A. Lendlein, J. Appl. Biomater. Funct. Mater., 2012, 10, 215-222.

32 A. Rosenhahn, S. Schilp, H. J. Kreuzer and M. Grunze, Phys. Chem. Chem. Phys., 2010, 12, 4275-4286.

33 C. Siegers, M. Biesalski and R. Haag, Chem.-Eur. J., 2004, 10, 2831-2838.

34 B. Seifert, G. Mihanetzis, T. Groth, W. Albrecht, K. Richau, Y. Missirlis, D. Paul and G. von Sengbusch, Artif. Organs, 2002, 26, 189-199.

35 J. G. Kelton and K. Steeves, Blood, 1983, 62, 924-927.
36 R. L. Nachman and A. J. Marcus, Br. J. Haematol., 1968, 15, 181-189.

37 M. Gawaz, F. J. Neumann and A. Schomig, Circulation, 1999, 99, E1-E11.

38 T. Cai, W. J. Yang, K.-G. Neoh and E.-T. Kang, Ind. Eng. Chem. Res., 2012, 51, 15962-15973.

39 Y. Chang, Y.-J. Shih, C.-Y. Ko, J.-F. Jhong, Y.-L. Liu and T.-C. Wei, Langmuir, 2011, 27, 5445-5455.

40 B. G. Keselowsky, D. M. Collard and A. J. García, Biomaterials, 2004, 25, 5947-5954.

41 P. Thevenot, W. Hu and L. Tang, Curr. Top. Med. Chem., 2008, 8, 270-280.

42 B. Sivaraman and R. A. Latour, Biomaterials, 2010, 31, 832839.

43 D. A. Herold, K. Keil and D. E. Bruns, Biochem. Pharmacol., 1989, 38, 73-76.

44 J. K. Armstrong, G. Hempel, S. Koling, L. S. Chan, T. Fisher, H. J. Meiselman and G. Garratty, Cancer, 2007, 110, 103111. 